\title{
Transcatheter ablation of arrhythmias associated with congenital heart disease
}

\author{
Tamas Szili-Torok • Laszlo Kornyei • Luc J. Jordaens
}

Received: 24 October 2007 / Accepted: 16 December 2007 /Published online: 5 March 2008

(C) The Author(s) 2008

\begin{abstract}
The improvement of surgical techniques resulted in significant life prolongation of many young patients with congenital heart disease (CHD). However, as these patients reach adulthood, their risk for late complications associated with surgery is also increased. One of the most difficult challenges associated with CHD is the high incidence of cardiac arrhythmias that arise from either the myocardial substrate created by abnormal physiology (pressure/volume changes, septal patches, and suture lines) or the presence of surgical scar. Catheter ablation is proven to be effective in treating atrial and ventricular arrhythmias in structurally normal hearts, and has also been used to treat arrhythmias in adults with congenital heart disease. In this review we provide an overview about diagnostic challenges, mapping and ablation techniques and outcome of patients undergoing transcatheter ablation procedures.
\end{abstract}

Keywords Catheter ablation - Congenital heart disease . Arrhythmias

T. Szili-Torok $\cdot$ L. Kornyei

Departments of Pacing and Clinical Electrophysiology,

Gottsegen Gyorgy Hungarian Institute of Cardiology,

Budapest, Hungary

T. Szili-Torok $\cdot$ L. J. Jordaens

Departments of Pacing and Clinical Electrophysiology,

Thoraxcentre, Department of Cardiology, Erasmus MC,

Rotterdam, The Netherlands

T. Szili-Torok $(\square)$

Gottsegen György Hungarian Institute of Cardiology,

Haller u. 29,

H-1096 Budapest, Hungary

e-mail: szili.torok@kardio.hu

\section{Background}

Arrhythmias associated with congenital heart disease encounter major diagnostic and therapeutic challenges for the treating physicians. Moreover, this very special patient group - often called GUCH (Grown Ups with Congenital Heart defects) - is followed-up in different clinical settings including pediatric cardiologists, congenital heart specialists, general cardiologists and rhythm-specialists-all depending on local organizations and the magnitude of the driving symptoms. Recurrent atrial tachycardias late after corrective or palliative surgery in patients with complex congenital heart disease (CHD) contribute considerably to the morbidity and mortality of this special group of patients [1]. Therefore, arrhythmia treatment is a key issue in the long-term outcome of these patients. All kind of arrhythmias can occur, and these arrhythmias are sometimes very challenging especially when only 12-lead ECGs are available. Based on the history of previous surgery and significant chamber dilatation, hypertrophy and fibrosis, atrial tachycardias in CHD patients often are macro reentrant tachycardias (MRAT) [1-5]. Certainly in many patients, atrial tachycardia has a focal mechanism. Viable myocardial fibers embedded within areas of scar tissue play a pivotal role in the initiation and perpetuation of macro reentrant tachycardias. Previous surgery and pathophysiological mechanisms involving the ventricles can cause ventricular arrhythmias. Catheter ablation may be a curative treatment for most atrial tachycardias in CHD patients and certainly might play a role in the treatment of ventricular tachycardias as well. The aim of the present review is provide about the most important diagnostic challenges and the most important approaches in the transcatheter ablative treatment of patients with CHD. 


\section{Epidemiology}

Patients with congenital heart disease (CHD) have an increased risk to develop arrhythmias [1, 5-7]. The high incidence of cardiac arrhythmias in the patients with CHD is either a result of coexisting congenital abnormalities of the specialized conduction system (congenital arrhythmogenic substrates) or may occur in response to hemodynamic influences on chamber dimensions, muscle mass, metabolism of myocardial tissue (acquired arrhythmogenic substrates) $[7,8]$. The best-known example of frequent association of congenital arrhythmogenic substrates with congenital defects is the accessory pathway (AP) associated with right-sided AV valve malformation. APs coexist in more than $25 \%$ of the patients with Ebstein anomaly [9]. Alterations in hemodynamics in CHD such as, chronic volume and pressure overload, cyanosis, myocardial hypertrophy and fibrosis, varying degrees of pulmonary hypertension and ventricular dysfunction all contribute to arrhythmogenesis.

Moreover, over the past few decades, surgical advances have helped to prolong the lives of many young patients with congenital heart disease but at the same time created the substrate for higher rate of acquired cardiac arrhythmias [7]. Septal patches, suture lines, surgical scars are superimposed on the myocardial substrate created by abnormal pressure/volume changes. Arrhythmias have been described as long-term complications after almost every form of cardiac surgery. However, certain combinations of CHD and surgical approaches seem to be associated with even higher rate of occurrence. These include surgeries involving an atriotomy or other significant surgical manipulation of the atrium, such as atrial septal defect repair, Mustard and Senning operations, Fontan anastomoses, tetralogy of Fallot and repair of anomalous pulmonary venous connection [7]. More than one quarter of patients who have undergone the Fontan and Mustard procedures have inducible sustained atrial tachycardias $[5,7,10,11]$.

\section{Diagnostic challenges}

The crucial point of creating the best strategy for ablation is to determine the right mechanism and the chamber of origin of tachyarrhythmias. In atrial tachycardia differentiating between a focal and reentry mechanism is often more difficult than in patients without structural heart disease. Obviously, micro-reentry tachycardias have a significant role in causing uncertainty in this process. Recently, a large number of algorithms became available including 12-lead ECG based approaches as well $[12,13]$.

There has been growing interest in elucidating the mechanism of atrial tachycardias and in locating the atrium of origin with the purpose of arrhythmia cure [13]. The surface ECG does not always provide good accuracy in predicting the chamber of origin. It is well known that even if the tachycardia demonstrates typical atrial flutter morphology, it still can be non-isthmus dependent. It is also known that even if the ECG is suggestive for FAT (focal atrial tachycardias), one may still have to deal with a MRAT (macro re-entrant atrial tachycardia). Eventually, the coexistence of more than one intra-atrial reentrant circuit cannot be excluded or predicted based on the 12-lead ECG alone [14]. For understanding the mechanisms of FATs an analogy can be drawn with normal impulse conduction. The sinus impulse for instance, which is more or less focal in origin, rarely takes more than $120 \mathrm{~ms}$ to spread over both atria when recorded with multipolar catheter configurations covering both atria. Regardless whether the pacemaker is the sinus node or an abnormal focus elsewhere in the atria, the activation front still spreads radially and it is rather hard to suppose that the time of the biatrial activation in FAT would be much longer than in normal sinus rhythm. Based on this, using two multipolar catheters in the heart (right atrium and coronary sinus) the biatrial activation recorded by these two catheters can serve as a basic measure to elucidate the mechanism of AT. Based on previous experience biatrial activation is almost always less than $40 \%$ of the whole tachycardia cycle length for FAT [13]. This can be used as a simple value for discrimination. Moreover, the ratio of RAA (right atrial activation) time related to TCL does not show any overlap between FAT and MRAT. It is known that in FAT arising from the LA close to the ostium of the right-sided pulmonary veins or at the superior part of the mitral annulus, the earliest atrial activation may be recorded in the posterior or septal area of the RA or at the terminal crest, and may precede the activation recorded at the CS. This is not surprising given the fact that the right superior pulmonary vein enters the LA just behind the superior cavoatrial junction and the right inferior pulmonary vein-behind the posterior RA wall midway between the superior and inferior cavoatrial junctions. As a matter of fact, the septal part of the RA is more leftward situated than the right lateral part of the LA where the pulmonary veins join it. This also explains the fact that in FAT, the earliest atrial activation taken in isolation as recorded by fixed position catheters is not a good predictor for the chamber of origin. It is known that in FAT originating from the left pulmonary veins, the activation of CS is from distal to proximal. If the direction of activation is proximal to distal, the focus can be within the RA or the LA near the right superior pulmonary vein. In MRAT, the activation front is circulating over a more or less large circuit from which it spreads out to the rest of the atria. When a multipolar catheter is deployed over the circuit or over a part of it, each electrode pair records the 
activation at different time points, which, taken together, constitute the TCL. If the catheter has enough electrode pairs with sufficient density, an atrial potential would be recorded virtually at every time point of TCL because the impulse is circulating continuously over the same path.

\section{Mapping techniques}

Inducibility, stability and the duration of the index arrhythmia determine the mapping possibilities in patients with CHD [15]. Obviously, a stable and sustained arrhythmia allows sequential data acquisition so as to perform activation mapping. This can be conventional activation mapping, 3D activation mapping and/or entrainment mapping $[6,13,15]$. Contrary to this, when the arrhythmia exhibits unstable activation sequences, or changing conduction times, mapping in sinus rhythm may be necessary. Mapping may also be useful after cardioversion in order to locate fixed barriers. In patients without inducible arrhythmia, a similar strategy can be useful.

\subsection{Entrainment mapping}

In atrial macroreentrant tachycardia, understanding the underlying reentrant circuit is the key to successful ablation $[6,16]$. Differentiating the right and left atrial origins of the tachycardia is of crucial importance for planning the procedure and for predicting the procedure's outcome and possible complications. Unfortunately, this determination may be difficult, especially if intra-atrial and interatrial conduction have already been altered by previous right and left atrial ablation, for example, after ablation for atrial fibrillation. Entrainment mapping is an established electrophysiologic technique that was first described in the 1980s and has been used since to delineate the localization of reentrant circuits. The most important criterion for determining whether a pacing site is located inside the reentrant circuit is the difference between the post-pacing interval (PPI) and the tachycardia cycle length (TCL). If the difference is 20 to $30 \mathrm{~ms}$, the pacing site probably is located inside the reentrant circuit. However, delineating a reentry using this technique can be laborious and timeconsuming because pacing from multiple sites is necessary.

\subsection{Three-dimensional electroanatomical mapping}

With the development of three-dimensional mapping systems, visualization of the entire electrical activation sequence during atrial reentrant tachycardia on the reconstructed anatomy of the right and left atria became possible [17]. Differentiation of the active atrium harboring the reentrant circuit from the passively activated atrium now is possible, but only after complete mapping of at least one atrium. Hence, a fast and easy way for distinguishing a right atrial from a left atrial origin of reentrant tachycardia is still lacking. Using 3D mapping as a single tool is therefore not recommended. Biatrial mapping improves diagnostic accuracy significantly. Furthermore, in a significant number of patients bi-atrial involvement is present, requiring bi-atrial ablation.

\subsection{Dual loop circuits}

The presence of more than one loop in postsurgical atria is the most challenging issue during transcatheter ablation procedures [18]. Dual-loop circuits should be anticipated and systematically approached in patients with IART late after open-heart surgery. The most important sign is sudden change of tachycardia CL and/or activation sequence during $\mathrm{RF}$ ablation. Tachycardia transformation is strongly suggestive for dual-loop IART but hard to notice in some cases [18]. Therefore reconfirmation using of entrainment mapping data at critical sites before catheter ablation and after tachycardia change aids in establishing the diagnosis. The circuits delineated so far have a distinctive pattern including an isthmus dependant atrial flutter and a periatriotomy circuit. Electroanatomic mapping appears useful to delineate the tachycardia isthmus of periatriotomy circuits.

\section{Transcatheter ablation of patients with the most common malformations}

\subsection{Arrhythmias after ASD repair}

The most common arrhythmias associated with ASD' are regular ATs and atrial fibrillation [7, 17, 19]. The transcatheter approaches to atrial fibrillation is beyond the scope of this review. For the treatment of AT different strategies are described. Three-dimensional electroanatomical mapping allows reconstruction of AT mechanisms and represents an advance in the precise localization and ablation of the arrhythmogenic substrate of post-surgical AT. Intraatrial macroreentrant mechanisms represented most of the mechanisms in most recent publications [20]. These are most often dependent on a narrow isthmus bounded by a line of double potentials related to the surgical incision. A much smaller number of patients develop tachycardias related to a focus of abnormal tissue adjacent to a right atrial incision or suture site. Ablation of both mechanisms can be performed with a high success rate.

\subsection{Ebstein's anomaly}

The deformity and displacement of the tricuspid valve can have serious hemodynamic consequences such as tricuspid 


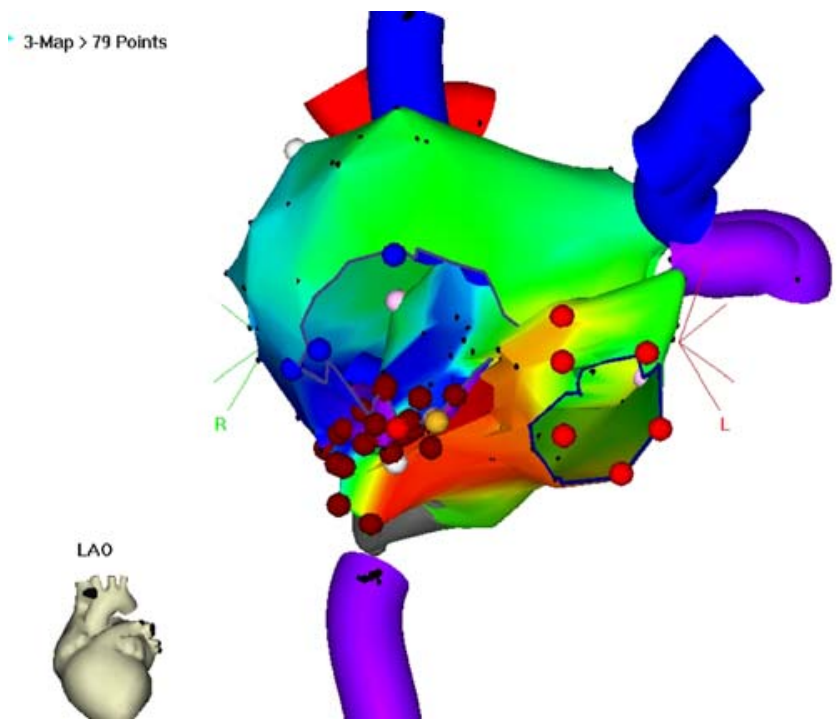

Fig. 1 CARTO 3D activation map showing bi-atrial activation map in a patient with atrial flutter associated with Senning operation. The activation shows cavotricuspid dependent atrial flutter with bi-atrial involvement

insufficiency and right atrial dilatation [9]. These abnormalities along with the frequent coexistence of atrial septal defects predispose to the development of arrhythmias. However, in this group of patients congenital arrhythmia substrates such as accessory pathways can make the spectrum very complex. Variants of accessory pathways such as Mahaim fibers are more common in this condition. Results of radiofrequency catheter ablation in Ebstein's anomaly for the treatment of AP and incisional MRAT are somewhat more disappointing than in patients without the anomaly. The factors contributing to this is clearly related to the complex geometry, location of the pathways in the atrialized portion of the right ventricle, the distorted $\mathrm{AV}$ ring and the presence of multiple accessory pathways.
Moreover, higher recurrences rates are also reported in patients with Ebstein's anomaly.

\subsection{Transposition of the great arteries: arrhythmias} after the Senning and Mustard operation

The Mustard and Senning operations were developed to correct the physiologic abnormality by forming a baffle within the atria to switch the flow of blood at the inflow level. The arterial switch operation was subsequently created to enable the left ventricle to become the systemic ventricle. The Mustard repair requires extensive incisions and suture lines in the atria. This results in intra-atrial conduction delays and abnormalities in atrial refractoriness, creating the substrate for atrial flutter $[11,16,21]$. Risk factors for supraventricular tachycardia include pulmonary hypertension, systemic ventricular dysfunction, and childhood junctional rhythm. Loss of sinus rhythm has been associated with previous septectomy, postoperative bradycardia, late atrial flutter, and preoperative arrhythmia. This loss of sinus rhythm is in contrast to the long-term maintenance of sinus rhythm in $95-98 \%$ of arterial switch patients. The incidence of supraventricular tachycardia is also significantly lower, with a rate of 5\% in arterial switch patients, compared to $48 \%$ in Mustard patients. Catheter ablation has been employed with a high acute success rate, and a moderate $10 \%$ rate of recurrence. The isthmus of tissue between the tricuspid valve and inferior vena cava orifice, the area of the mouth of the coronary sinus, and the region extending from the mouth to the tricuspid annulus are critical components in the reentry circuit. Intra-atrial reentry may involve either atrium and requires a retrograde aortic approach in most cases leading to biatrial ablation. Even cavotricuspid isthmus ablation requires bi-atrial
Fig. 2 Ablation of Figure 8 type dual loop macroreentry in a patient long after the correction of tetralogy of Fallot. The left panel shows the activation map with the ablation points (red dots), while the right panel demonstrates integration with the magnetic navigation system
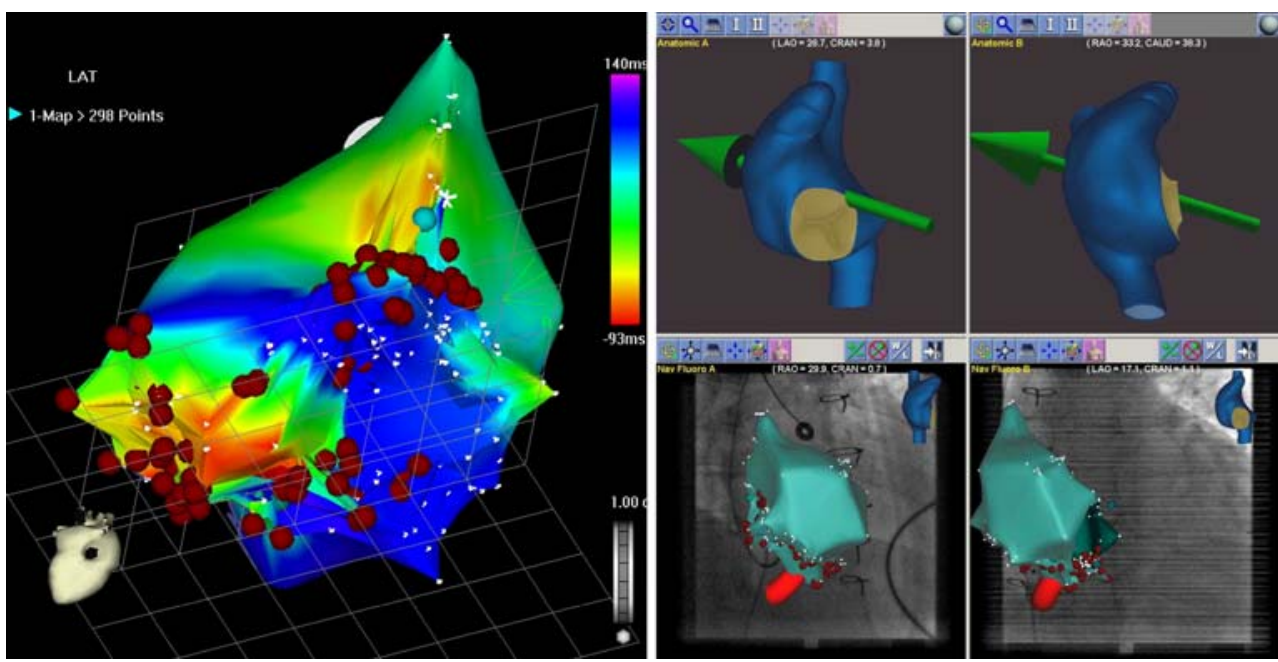
ablation in most patients (Fig. 1). In addition, focal atrial tachycardia has been localized adjacent to baffle suture lines.

\subsection{Tetralogy of Fallot}

Patients with this condition are predisposed to both ventricular and atrial arrhythmias [1, 3, 10, 22, 23]. Therefore, the differential diagnosis is a crucial step in the early management of the patients. Ventricular arrhythmias have a prevalence of $4-7 \%$ and the 12-lead ECG usually shows right ventricular outflow tract type morphology [23, 24]. On top of this, more than one third of TOF patients manifest atrial arrhythmias [3]. Risk factors include older age at operation, increased atrial size, tricuspid or pulmonary regurgitation and ventricular dysfunction. Atrial flutter and atrial fibrillation were more common in patients who had long-lasting pulmonary artery shunts, early operations for residual hemodynamic lesions, older age at repair, and moderate-to-severe tricuspid regurgitation [25]. Catheter ablation is successful in patients with atrial flutter and VT. A scar tissue caused by the corrective surgery should be identified for successful ablation. An ablation line between the lower margin of the scar and the inferior vena cava is associated with a high success rate, with a moderate recurrence rate (Fig. 2). VT's are usually localized to the right ventricular outflow tract and the infundibulotomy scar or the septal surface of the VSD patch repair. Interestingly enough, beyond the high immediate success rate, these procedures are associated with very low rate of recurrences [4].

\subsection{Fontan circulation and ablation procedures}

Older variants of the Fontan procedure such as the right atrium-pulmonary artery connection, have given way to newer modifications such as the lateral tunnel and external conduit, which reduce the distention of the right atrium $[5,26]$. In response to chronic stretching secondary to persistent pressure overload, the Fontan right atrium remodels and dilates [27]. This occurs in parallel to the change in electrophysiological properties manifested by the atrial conduction delay and the imbalance of the conduction heterogeneity. There are certain risk factors for early postoperative arrhythmias such as atrioventricular $\mathrm{AV}$ valve regurgitation. Older age at operation is also a risk factor in almost all congenital malformations [27]. Atrial arrhythmias develop in a very high percentage of patients. Patients who develop atrial arrhythmias have more severe co-morbidities including heart failure, and right atrial thrombus. CA is associated with a surprisingly high acute success rate of more than $80 \%$ with a relatively high $(20 \%)$ incidence of recurrences. The most common arrhythmia substrates are located in the region of the Fontan anastomosis, the lateral right atrial wall and the inferior right atrium [28].

\section{The possible role of novel navigation technologies in catheter ablation of $\mathrm{CHD}$ patients}

Developments allowing better navigation and 3D image integration may improve outcome of catheter ablation procedures in GUCH patients. One of the most promising technologies is magnetic navigation (Fig. 2). There are theoretical advantages of using magnetic navigation applicable for these complex and challenging anatomical situations. One of the potential advantages of the system is the utilization of a non-traumatic, very floppy catheter. It reduces the risk for perforation, and it can easily pass fenestrations associated with previous surgery. Another advantage is the superior navigation capability. Certainly, increased stability might provide better transmural lesions as well. The combination of navigation and $3 \mathrm{D}$ imaging such as CT, MRI and ultrasound will further improve success rate and theoretically can play a role in decreasing arrhythmia recurrences.

\section{Conclusions}

Atrial tachycardia in patients with surgically corrected CHD is usually caused by a macroreentrant mechanism. However, atrial tachycardia is caused by a focal mechanism in a considerable number of patients. Moreover exact determination of the chamber of origin is essential for the appropriate ablation strategy. Areas of abnormal conduction may be the crucial pathway within a large reentrant circuit, but they also may be the site of origin of a focal atrial tachycardia and thus play an important role in the pathogenesis of these atrial tachycardias. Catheter ablation directed at areas of abnormal conduction ("source ablation") is mostly effective in these patients. Novel guiding and mapping tools improve the efficacy of ablation, however full integration with catheter technology is still an important issue to be solved.

Open Access This article is distributed under the terms of the Creative Commons Attribution Noncommercial License which permits any noncommercial use, distribution, and reproduction in any medium, provided the original author(s) and source are credited.

\section{References}

1. Li, W., \& Somerville, J. (2000). Atrial flutter in grown-up congenital heart $(\mathrm{GUCH})$ patients. Clinical characteristics of affected population. International Journal of Cardiology, 75(2-3), 129-37 discussion 38-9.

2. Hansen, D. E., Craig, C. S., \& Hondeghem, L. M. (1990). Stretchinduced arrhythmias in the isolated canine ventricle. Evidence for the importance of mechanoelectrical feedback. Circulation, 81(3), 1094-1105. 
3. Roos-Hesselink, J., Perlroth, M. G., McGhie, J., \& Spitaels, S. (1995). Atrial arrhythmias in adults after repair of tetralogy of Fallot. Correlations with clinical, exercise, and echocardiographic findings. Circulation, 91(8), 2214-2219.

4. Rostock, T., Willems, S., Ventura, R., Weiss, C., Risius, T., \& Meinertz, T. (2004). Radiofrequency catheter ablation of a macroreentrant ventricular tachycardia late after surgical repair of tetralogy of Fallot using the electroanatomic mapping (CARTO). Pacing and Clinical Electrophysiology, 27(6 Pt 1), 801-804.

5. Warnes, C. A., \& Somerville, J. (1986). Tricuspid atresia in adolescents and adults: current state and late complications. British Heart Journal, 56(6), 535-543.

6. Walsh, E. P. (2007). Interventional electrophysiology in patients with congenital heart disease. Circulation, 115(25), 3224-3234.

7. Balaji, S., \& Harris, L. (2002). Atrial arrhythmias in congenital heart disease. CardiologiÂa cliÂnica, 20(3), 459-68 vii.

8. Magnin-Poull, I., De Chillou, C., Miljoen, H., Andronache, M., \& Aliot, E. (2005). Mechanisms of right atrial tachycardia occurring late after surgical closure of atrial septal defects. Journal of Cardiovascular Electrophysiology, 16(7), 681-687.

9. Younoszai, A. K., Brook, M. M., \& Silverman, N. H. (1999). Ebstein's Malformation. Current Treatment Options in Cardiovascular Medicine, 1(4), 363-372.

10. Atik, F. A., Atik, E., da Cunha, C. R., et al. (2004). Long-term results of correction of tetralogy of Fallot in adulthood. European Journal of Cardio-Thoracic Surgery, 25(2), 250-255.

11. Kriebel, T., Tebbenjohanns, J., Janousek, J., Windhagen-Mahnert, B., Bertram, H., \& Paul, T. (2002). Intraatrial reentrant tachycardias in patients after atrial switch procedures for d-transposition of the great arteries Endocardial mapping and radiofrequency catheter ablation primarily targeting protected areas of atrial tissue within the systemic venous atrium. Zeitschrift fuÉr Kardiologie, 91(10), 806-817.

12. Kistler, P. M., Roberts-Thomson, K. C., Haqqani, H. M., et al. (2006). P-wave morphology in focal atrial tachycardia: development of an algorithm to predict the anatomic site of origin. Journal of the American College of Cardiology, 48(5), 10101017.

13. Shalganov, T. N., Vatasescu, R., Paprika, D., et al. (2006). A simple algorithm for defining the mechanism and the chamber of origin in atrial tachycardias. Journal of Electrocardiology, 39(4), 369-376.

14. Deisenhofer, I. (2006). Simplified algorithm for localization of atrial macroreentrant tachycardias: keep it simple and short. Heart Rhythm, 3(5), 524-525.

15. Pflaumer, A., Deisenhofer, I., Hausleiter, J., \& Zrenner, B. (2006). Mapping and ablation of atypical flutter in congenital heart disease with a novel three-dimensional mapping system (Carto Merge). Europace, 8(2), 138-139.
16. Akar, J. G., Kok, L. C., Haines, D. E., DiMarco, J. P., \& Mounsey, J. P. (2001). Coexistence of type I atrial flutter and intra-atrial reentrant tachycardia in patients with surgically corrected congenital heart disease. Journal of the American College of Cardiology, 38 (2), 377-384.

17. Triedman, J. K., Alexander, M. E., Berul, C. I., Bevilacqua, L. M., \& Walsh, E. P. (2001). Electroanatomic mapping of entrained and exit zones in patients with repaired congenital heart disease and intra-atrial reentrant tachycardia. Circulation, 103(16), 20602065.

18. Seiler, J., Schmid, D. K., Irtel, T. A., et al. (2007). Dual-loop circuits in postoperative atrial macro re-entrant tachycardias. Heart, 93(3), 325-330.

19. Cosio, F. G., Martin-Penato, A., Pastor, A., Nunez, A., \& Goicolea, A. (2003). Atypical flutter: a review. Pacing and Clinical Electrophysiology, 26(11), 2157-2169.

20. Bottio, T., Leoni, L., Vida, V., Stellin, G., Casarotto, D., \& Gerosa, G. (2003). Radiofrequency ablation through a right atrium incision in congenital atrial septal defect. Langenbeck's Archives of Surgery, 388(1), 52-55.

21. Giamberti, A., Chessa, M., Abella, R., et al. (2007). Surgical treatment of arrhythmias in adults with congenital heart defects. International Journal of Cardiology (in press), August 7.

22. Dietl, C. A., Cazzaniga, M. E., Dubner, S. J., Perez-Balino, N. A., Torres, A. R., \& Favaloro, R. G. (1994). Life-threatening arrhythmias and RV dysfunction after surgical repair of tetralogy of Fallot. Comparison between transventricular and transatrial approaches. Circulation, $90(5 \mathrm{Pt}$ 2), II7-II12.

23. Folino, A. F., \& Daliento, L. (2005). Arrhythmias after tetralogy of Fallot repair. Indian Pacing Electrophysiol J, 5(4), 312-324.

24. Harrison, D. A., Harris, L., Siu, S. C., et al. (1997). Sustained ventricular tachycardia in adult patients late after repair of tetralogy of Fallot. Journal of the American College of Cardiology, 30(5), 1368-1373.

25. Gatzoulis, M. A., Balaji, S., Webber, S. A., et al. (2000). Risk factors for arrhythmia and sudden cardiac death late after repair of tetralogy of Fallot: a multicentre study. Lancet, 356(9234), 975981.

26. Deal, B. J., Mavroudis, C., \& Backer, C. L. (2003). Beyond Fontan conversion: Surgical therapy of arrhythmias including patients with associated complex congenital heart disease. Annals of Thoracic Surgery, 76(2), 542-553 discussion 53-4.

27. Weipert, J., Noebauer, C., Schreiber, C., et al. (2004). Occurrence and management of atrial arrhythmia after long-term Fontan circulation. Journal of Thoracic and Cardiovascular Surgery, 127 (2), 457-464

28. Agnoletti, G., Borghi, A., Vignati, G., \& Crupi, G. C. (2003). Fontan conversion to total cavopulmonary connection and arrhythmia ablation: clinical and functional results. Heart, 89(2), 193-198. 